\title{
Development and evaluation of a Web-based e-book with a concept mapping system
}

\author{
Liang-Yi $\mathbf{L i}^{1}$
}

Received: 30 October 2014/Revised: 15 March 2015/Accepted: 16 March 2015/

Published online: 27 March 2015

(C) Beijing Normal University 2015

\begin{abstract}
This paper describes the development of a Web-based e-book with a concept mapping system and the process and results of a concept mapping course in which 139 seventh-grade students used the developed system. Two iterated system development cycles were completed. The second system prototype provides an e-book system and a concept mapping system on the same Web page. Users can read an article and construct a concept map for the article simultaneously. Concept mapping course was conducted during a 3-week period. The results of the course showed that the students demonstrated a positive attitude toward concept mapping after using the system in the course. However, the quality of their concept maps is low. Several mistakes that the students frequently made were explored. In addition, the analysis of the students' annotation behaviors showed that some students use text highlight to support their construction of concept maps. However, an independent-sample $t$ test did not demonstrate a significant effect of text highlight on the quality of concept maps. These results provide directions for our future work. Furthermore, several requirements are summarized in this paper according to the feedback of the teacher and students. These requirements are the base for our next iterative system and course design.
\end{abstract}

Keywords Concept map $\cdot$ e-Book $\cdot$ Annotation $\cdot$ Reading strategy

This manuscript is an extended version of GCCCE2014 paper "The Development and Evaluation of a Web-Based Concept Mapping System for Supporting Reading”.

Liang-Yi Li

lihenry12345@gmail.com

1 Research Center for Science and Technology for Learning, National Central University, No. 300, Jhongda Rd., Jhongli City, Taoyuan County 32001, Taiwan, ROC 


\section{Introduction}

Reading comprehension is an interactive process between the reader and the text. Readers simultaneously extract and explain the written text and integrate it with their prior knowledge (Al-Khateeb and Idrees 2010; Kintsch 1998). Reading strategies, which play a vital role in reading comprehension, have been recognized as effective approaches in increasing reading comprehension. Several strategies have already been proven to support reading comprehension, such as note-taking, questioning, and graphic organizers (Huang et al. 2009).

Graphic organizing is a common reading strategy and plays two roles in reading comprehension: encoding and reviewing. Identifying main ideas in a text is essential to reading comprehension. Graphic organizers enable readers to identify the main ideas in a text and the relationship between them and to encode them into a coherent structure. This process helps readers remember and comprehend the text. In addition, reviewing a well-organized two-dimensional structure improves recall and comprehension compared with reading linear text.

Many graphic organizers, including the mind map, concept map, knowledge map, and argument map, have been developed. These graphic organizers appear similar; however, their purposes and structures as well as the relationships among nodes are different (Davies 2011). Concept maps are frequently used in academic disciplines. It is a node-link diagram, which represents a concept using a node and a relationship using a link (Novak and Gowin 1984). The aim of concept mapping is to outline relationships between ideas. A concept map has a hierarchical "tree" structure with superordinate (general concepts) and subordinate (specific concepts) parts. Learners identify the concepts in a text, arrange the concepts, and associate them with linking words when constructing a concept map (Novak 2010; Novak and Gowin 1984).

Computerized concept mapping systems have been developed with the advancement of Information Communication Technology (ICT). Compared with the traditional approach, which involves using paper and pens, computerized concept mapping has several advantages. For example, students can more easily construct, modify, and maintain their concept maps. The concept nodes can be associated with external Internet resources (Weinerth et al. 2014). In addition, the World Wide Web (WWW) enables people to access Web applications and content at any time and location. This increases the mobility and interoperability of concept mapping because only a Web browser is required to access concept mapping applications. Therefore, some websites have provided Web-based concept mapping tools for people to use, share, and store concept maps.

Although the WWW provides numerous advantages for concept mapping, it has two major difficulties for developing Web applications that require complex operation and intensive interaction, such as concept mapping. First, the program and data on the client side (HTML, JavaScript, and CSS) must be coordinated with those on the server side (PHP, JSP, and ASP.Net). The complexity of Web programming is higher than that of window programming. Second, the components and interactions of the graphic user interface (GUI) are more difficult to implement in Web programming than in window programming. Although several HTML5 
JavaScript UI libraries, such as jQuery UI (http://jqueryui.com/), Kendo UI (http:// www.telerik.com/), and Ext JS (http://www.sencha.com/), have been developed, they provide limited GUI components and interactions. Therefore, the first purpose of this study was to develop a Web-based concept mapping tool and to share our experiences in developing it.

Teachers often combine the concept mapping strategy with reading activities. The recent development of e-ink and tablet technologies has popularized reading with digital devices. These technologies provide novel functionalities to reduce the drawbacks of reading on screen. Furthermore, the ICT provides certain advantages for teaching and learning, such as flow monitoring and controlling, knowledge sharing, and information visualization. Integrating these advantages into the reading process facilitates reading comprehension. Therefore, determining how to integrate reading strategies into e-books is a crucial research topic. The second purpose of this study was to develop a Web-based e-book system and to integrate it into the Web-based concept mapping tool.

The Web-based e-book system with a concept mapping tool is innovative. Users' attitude, behavior, and learning outcome are unknown. Therefore, the third purpose was to conduct an experimental course to collect users' feedback on the system and the concept mapping course and to examine users' attitude, behavior, and learning outcome to the system and course.

\section{Related work}

\section{Computerized concept mapping tools}

Numerous computerized concept mapping tools, such as IHMC CmapTools (Novak and Cañas 2008), TPL-KATS (Hoeft et al. 2003), concept mapping systems (Chang et al. 2001), CMKMS (Liu and Lee 2013), Easy Mapping Tool (http://www. cognitive-tools.com/), CM-ED (http://galan.ehu.es/Galan/products), and concept mapping software programs (Liu et al. 2010), have been developed. These tools enable construction of any type of diagram. They provide an excellent humancomputer interface and interaction, enabling users to learn efficiently and complete concept mapping tasks effectively. However, they require users to install the programs onto their personal computers, limiting mobility and interoperability.

In addition to computerized concept mapping tools, several Web systems such as the Web Version Mapping Tool (http://www.cognitive-tools.com/), Lucidchart (https://www.lucidchart.com/), Cacoo (https://cacoo.com/getstarted/mappingtools), and Webspiration (http://www.webspirationpro.com/) provide functions for creating and maintaining diagrams. Users can use these systems to create concept maps on the Internet, improving mobility. However, some of these systems require users to install extra plugins. For example, the Web Version Mapping Tool was developed in the Java programming language. Browsers must therefore have the Java virtual machine installed in order to interact with the system. Similarly, Cacoo and Webspiration were developed using Flash. These plugins cannot be operated in some mobile operating systems, limiting interoperability. 
This study referred to the aforementioned concept mapping tools in developing a Web-based concept mapping system. The system was implemented in JavaScript programming language, which can be operated in any device and browser without requiring extra plugins, improving mobility and interoperability.

\section{E-book systems with reading strategies}

Reading from a screen has become increasingly popular in recent years. Users can read text on various devices such as laptops, mobile phones, and tablets. These devices provide e-book systems for users to interact with digitalized text. Most e-book systems provide basic functions that support reading, such as highlighting, commenting, searching, and tagging. To enhance reading comprehension, several studies have integrated reading strategies into e-book systems. For example, Li et al. (2014) integrated the SQ3R reading strategy into an e-book system. Kozminsky (2010) and Ponce et al. (2012) have incorporated graphic organizers into e-book systems. van der Pol et al. (2006) integrated a collaborative discussion activity into an e-book design.

Concept mapping is frequently combined with reading. Although the aforementioned e-book systems have been integrated with reading strategies, we did not find any study that described the methods for designing and developing an e-book system with concept mapping.

\section{System development}

System development is an iterative process that involves design, implementation, and evaluation. In the present state of system development, we have completed two cycles of this process.

\section{First development cycle}

In the first cycle, we developed the first system prototype on the basis of the systems introduced in "Related work" section. The first prototype consisted of an e-book system, which provided highlighting and commenting functions, and a concept mapping system, which enabled users to create, modify, and delete nodes and links. After implementing the prototype, we invited six second-year graduate students to read a book related to critical thinking. We selected six chapters and inserted them into our e-book system. The students were required to read one chapter (each chapter was approximately 10 pages) per week for 6 weeks. During the 6-week period, we occasionally interviewed the students to collect feedback on the system. Their feedback is listed as follows:

(1) I hope the system can provide more colors for the nodes and links (the first prototype had only a white background, black text for nodes, and black linking lines).

(2) I hope the system can let me save the concept map at different time points and return to the concept maps saved at those time points (the first prototype saved only the most recent version of the concept map). 
(3) I hope the system can let me move multiple nodes simultaneously (the first prototype enabled only one node to be moved at a time).

(4) I hope the system can provide more colors and styles for text marking (the first prototype provided only blue highlighting).

(5) I hope the system can allow me to ask questions and engage in discussion with my classmates.

(6) I hope the e-book and concept mapping interface can be displayed on the same page so that I do not need to switch between them (the first prototype displayed the two interfaces on separate pages).

\section{Second development cycle}

In the second cycle, we designed the system according to the feedback. All requests except the fourth request have been satisfied. After implementing the second prototype, we conducted an experimental course to collect additional user feedback on the system to understand how users operate the system and their preferences and learning outcomes from the concept mapping course. "System description" section describes the second prototype. "Feedback on the concept mapping course" section discusses the results of the experimental course.

\section{System description}

The second prototype consists of three sections: e-book, concept mapping, and function sections (Fig. 1). The e-book section provides the e-book content. The concept mapping section enables users to construct and maintain a concept map.

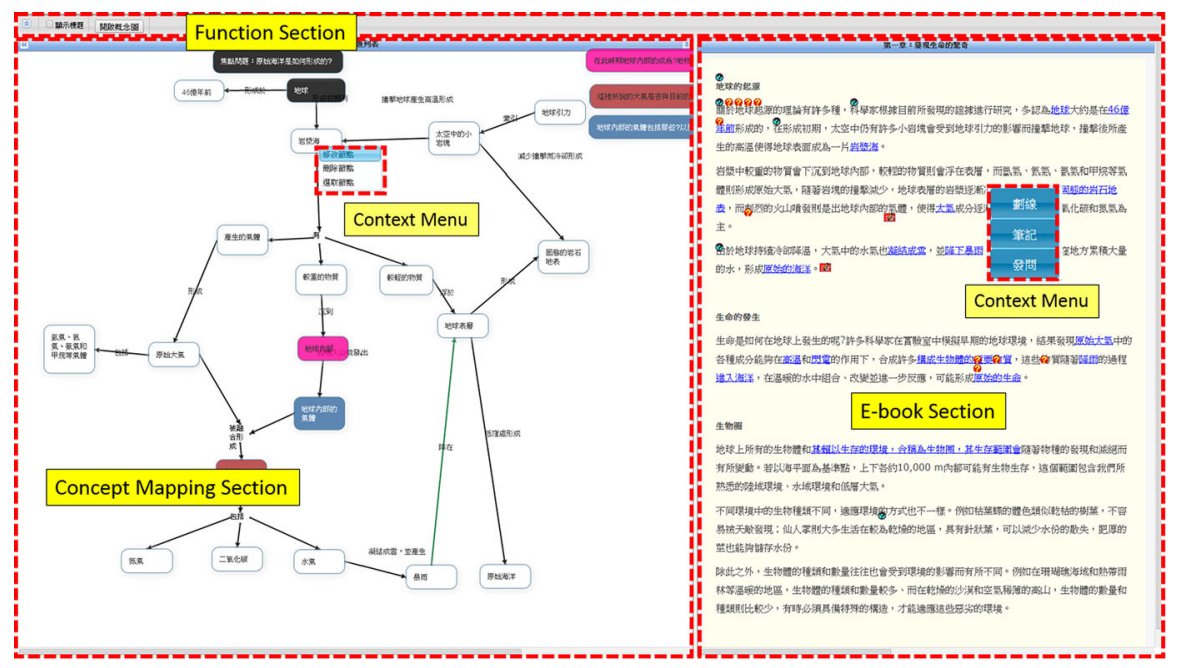

Fig. 1 Interface of the second prototype 
The function section provides several buttons for interacting with the e-book content.

In the e-book section, users can read text while using the highlight, comment, and question functions on any text. When a text segment is marked, a menu is immediately displayed. The menu consists of three items: "Highlight", "Comment", and "Question". A user can click "Highlight" to immediately highlight the marked text segment. When the user clicks "Comment", a comment form appears for him or her to enter a comment. When a comment is entered, a comment icon appears above the marked text segment. When the user clicks "Question", a question form appears for him or her to enter a question. When a question is entered, a question icon appears above the marked text segment. All students in the same class can see the question icon in their e-book and reply to the question.

Users can click on a highlighted text segment or a comment icon using the right mouse button to delete the highlight or comment. Because questions are shared among all students, they cannot be deleted. Users can also use the left mouse button to click on a comment icon to edit the comment or on a question icon to reply to the question. Comment and question icons can be dragged to any place in the document. Question icons can be in blue or red. Blue indicates that the student had read the question; red indicates that the question is unread.

Users can construct a concept map for the text in the concept mapping section. To create a new node in a location, a user can click on the position using the left mouse button to access the context menu, which consists of two menu items, "Create Node" and "Create New Version". When the user clicks "Create Node", a form appears for him or her to enter a title and description and to select a text color and background color for the node. After the form is completed, a node is generated with the chosen features in the selected position. When the user clicks "Create New Version", the system immediately saves the current concept map with a version number. The user can return to any version number in the concept map management interface. When clicking on any node, he or she can edit or delete it. The user can also drag any node or select multiple nodes and move them to any location.

When the user's mouse hovers over a node, the node immediately shows a small red square. The user can drag a line from the square and drop it to another node. When the user drops the line on a node, a form is shown asking him or her to enter linking words and select a line color and an arrow. A link with the linking words, line color, and arrow is then constructed between the two nodes.

Two buttons are displayed in the function section: "Open Concept Map" and "Show Title". When a user clicks "Open Concept Map", the concept map is immediately displayed on a new page. Compared with the concept mapping section, displaying in a new page provides more space for the user to draw his or her concept map. When the user clicks "Show Title", the titles of comments and questions are shown adjacent to the respective comment and question icons, enabling the user to view the titles of all comments and questions.

The system is a three-tier structure comprising the client, server, and database. In the client program, we used HTML, JavaScript, and JQuery UI to provide the interface and control interaction. All data created by users are sent to the server in JavaScript Object Notation format using Asynchronous JavaScript and XML. The 
server program was written using a Java servlet and Java server page. The server program receives the data sent from the client program and performs database operation; the results are then sent to the client. The client program receives the results and modifies components in the interface. We used Apache Tomcat 7.0 as the Web server and MySql as the database server. Most of the GUI components for implementing this system can be found in JQuery UI. The most complex part of the design is the interface for and interaction in concept mapping. However, JavaScript libraries provide diagram components and methods for creating and maintaining concept maps, such as jsPlumb (http://www.jsplumb.org/) and GoJS (http://www. nwoods.com/). Programmers can use these libraries to develop a Web-based concept mapping system.

\section{Evaluation}

After implementing the second prototype, we conducted an experimental course. Three classes participated in the course. Classes A, B, and C had 47, 46, and 46 students, respectively. They were seventh-grade students. This course would like to collect the feedback on the Web-based e-book system and concept mapping instruction. Except collecting users' feedback, this course also collected data to answer the following three questions.

1. What is the students' attitude toward the concept mapping?

2. What is the learning outcome of the students after using the system in the course?

3. How did the students use the system to complete a concept map?

The same teacher taught all three classes. The teacher conducted the course for $2 \mathrm{~h}$ per week for 3 weeks for each class in a computer classroom. All students used the Google Chrome browser to interact with our system. In the first week, the teacher described how to construct a concept map and how to use our system in the first hour. Concept mapping instruction was conducted according to the suggestions provided by Novak and Gowin (1984). All of the instruction sessions were recorded on video. The students watched the videos to learn how to construct a concept map ( 5 min and $35 \mathrm{~s}$ ) and how to use this system (6 min and $2 \mathrm{~s}$ ). The teacher then gave the students an article (61 Chinese characters) and asked them to construct a concept map for the article. If they encountered difficulty, they could watch the videos again or ask the teacher for support. After the students completed the concept map, the teacher selected the concept maps of two students and explained to all students how to improve the maps.

Because students who collaboratively construct concept maps can learn from each other and create higher quality maps (Kwon and Cifuentes 2009), the teacher divided the students into groups of two (23 groups per class). In the second hour, the teacher gave each group an article (114 Chinese characters) and asked them to collaboratively construct a concept map for the article (Fig. 2). The students could 
ask the teacher questions if they encountered problems when constructing their concept map.

In the first hour of the second week, the teacher explained again to all the students the essential points for constructing a concept map, described the common mistakes that the students made in their concept maps for the second article, and explained how to correct the mistakes. The students then corrected their concept maps according to the teacher's suggestions.

In the second hour, the teacher gave the students a third article (293 Chinese characters) and asked them to collaboratively construct a concept map for the article.

In the first hour of the third week, the teacher described to all the students the common mistakes that they made in their concept maps for the third article and explained how to correct the mistakes. The students then corrected their concept maps according to the teacher's suggestions.

In the second hour, the teacher administered the students a questionnaire that consisted of 18 questions (12 single-choice questions and six open-ended questions). The single-choice questions were ranked on a 5-point Likert scale and were used to understand the students' self-efficacy in learning concept mapping (1-6), intention to use concept mapping (7-8), and attitude toward concept mapping (9-12). The open-ended questions generated feedback on the system and course and are listed as follows:

(1) What are your suggestions on the instruction process for this course?

(2) What are your suggestions on the content of this course?

(3) What are your suggestions on the videos used in this course?

(4) What are your suggestions on the e-book system?

(5) What are your suggestions on the concept mapping system?

(6) Do you have any other suggestions?

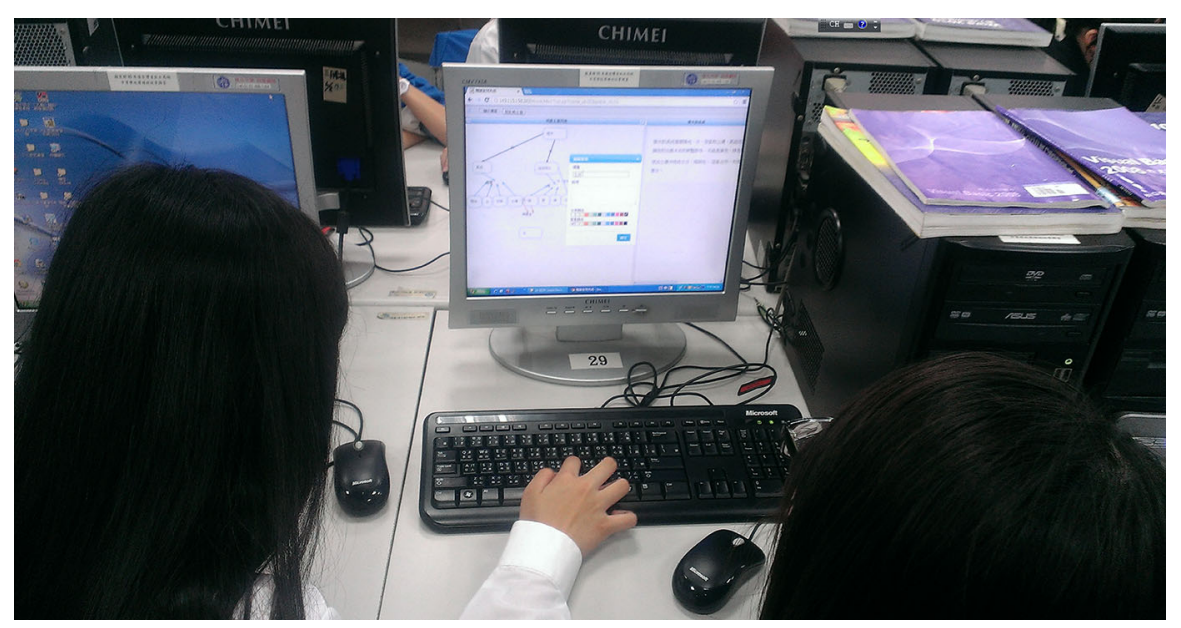

Fig. 2 Students worked in pairs to construct a concept map 


\section{Results}

\section{Feedback on the e-book and concept mapping system}

In the questionnaire, the students proposed advanced functions for the system. We summarized their suggestions as follows:

(1) Provide different styles for linking lines, such as curved lines or dotted lines (the linking line in the second prototype is a solid straight line) (38/139).

(2) Provide different colors and styles for text marking (this suggestion was also proposed for the first prototype) (23/139).

(3) Provide more colors for linking lines and nodes (the second prototype provided only 13 colors) (13/139).

(4) Enable the posted questions to be edited and deleted (12/139).

(5) Provide "undo" and "redo" functions (11/139).

(6) Enable the nodes to align automatically (10/139).

(7) Provide more styles for the text font and format (9/139).

Suggestions (4), (5), and (6) are functional. Regarding suggestion (4), if a student edits or deletes a question, then other students who have replied to the question may feel disrespected or confused about the discussion. To solve this problem, perhaps the questions that have not been replied to can be deleted or edited by the author, and the students can also ask their teacher to edit or delete posts if the teacher thinks that it is suitable. We think that suggestions (5) and (6) are necessary and will implement them in the next development cycle.

Suggestions (1), (2), (3), and (7) were related to providing more styles and formats. The suggested improvements may have several purposes. First, students can use different colors to organize information. For example, they can use different colors to highlight text segments that have different meanings for them. They also can use different colors for nodes with different hierarchical levels. Second, they may feel that learning tasks are more engaging and fun if they can use more colors and styles. Third, they may desire more control in the system. Therefore, providing more flexibility and control for the user and more styles for presenting information is crucial for designing human-computer interfaces and interaction. However, students might spend more effort and time thinking about how to style their concept maps than they do in constructing them.

The teacher suggested providing an authoring tool in the system for several concept mapping tasks. The tasks consist of (1) correcting an erroneous concept map, (2) completing a partial concept map, (3) completing a partial and erroneous concept map, (4) providing a list of concepts and linking words for students to construct a concept map, and (5) providing a list of concepts and linking words for students to complete a partial and erroneous concept map. Previous studies have proposed these tasks and found that they help students learn concept mapping. However, these studies did not present their authoring tools in their papers. The interface of the authoring tools used in these studies is unknown. 


\section{Feedback on the concept mapping course}

The students also provided several suggestions regarding the process and content of the experimental course. The most frequently provided suggestions are listed as follows:

(1) The content in the videos can be explained by a human voice with subtitles instead of only by subtitles (33/139).

(2) The content and process of the course should be fun and engaging (32/139).

(3) The teacher or the videos can demonstrate more examples on how to construct a concept map (12/139).

(4) The teacher can provide a variety of interesting reading materials instead of only science articles (11/139).

(5) The teacher can give students more time to discuss and construct a concept map (9/139).

(6) The teacher can demonstrate how to construct a concept map for a complex article step by step (9/139).

(7) The teacher can engage in more discussion and interaction with students (8/ 139).

Suggestions (3) and (6) are related to proving modeling scaffolding support. The instructional videos solely demonstrate how to construct a concept map for a simple article (first article). However, the third article is more complex. The students may need more support for identifying concepts, links, and proposition. The students also hope that the instruction can be fun and engaging (Suggestions 2, 4, and 7).

In addition to the students' feedback, the teacher also had two comments on the concept mapping course. First, the 3-week instruction duration was not sufficient. Students should be trained for a longer duration or given more time to construct the maps. Second, because most courses do not have extra time to teach students reading strategies, concept mapping should be incorporated into course activities and content.

\section{Students' attitude toward concept mapping}

Table 1 lists the results of the questionnaire. The results of the single-choice questions showed that the students had a positive attitude toward concept mapping. They believed that concept mapping can help them remember and comprehend text and, thus, improve their learning outcome. They also believed that they are capable of constructing a concept map for an article after the 3-week training course. However, the mean of intention to use concept mapping was low.

\section{Analysis of the quality of the students' concept maps}

Because the contents of the third article are more complex and the students were already very familiar with the system and the construction of a concept map when reading the article, we just analyzed the concept maps of the third article. To 
Table 1 The results of the questionnaire related to self-efficacy, intention to use, and attitude toward concept mapping

\begin{tabular}{llcc}
\hline & Question items & Mean & SD \\
\hline 1 & I am capable of identifying the concepts in an article & 4.23 & 0.75 \\
2 & I am capable of identifying which concepts are superordinate and which are & 4.24 & 0.75 \\
& & \\
& $\quad$ subordinate & 4.22 & 0.80 \\
3 & I am capable of finding the relationship between concepts & 4.13 & 0.71 \\
4 & I am capable of integrating two or three concepts into a proposition & 4.09 & 0.90 \\
5 & I am capable of finding the crosslinks & 4.08 & 0.92 \\
6 & I am capable of constructing a concept map for an article & 3.74 & 1.02 \\
7 & I intend to use concept mapping in my future study & 3.81 & 0.97 \\
8 & I will use concept mapping in other courses & 4.09 & 0.85 \\
9 & Concept mapping helps me to comprehend the text & 4.12 & 0.89 \\
10 & Concept mapping help me easily recall the text & 4.17 & 0.86 \\
11 & I believe that I can have a better learning outcome through concept mapping & 4.04 & 0.88 \\
12 & I believe that I can achieve my learning goals through concept mapping & 4.04 \\
\hline
\end{tabular}

evaluate the quality, the scoring criteria referred to Novak and Gowin (1984) and Tzeng (2014). The scoring rules are listed as follows:

1. A node is properly annotated (2 points).

2. A link can validly connect two nodes, and the nodes are properly annotated (2 points).

3. A link can validly connect two nodes, but the nodes are not properly annotated (1 point).

4. A link is not properly annotated ( -1 point).

5. The associated propositions are properly clustered (10 points).

6. A cross link can validly connect two nodes between different clusters (5 points).

Table 2 The analysis of the students' concept maps

\begin{tabular}{llllr}
\hline & Min & Max & Mean & Expert \\
\hline Number of nodes & 1 & 31 & $20.29(\mathrm{SD}=5.52)$ & 23 \\
Number of links & 0 & 38 & $21.17(\mathrm{SD}=7.12)$ & 26 \\
$\begin{array}{l}\text { Number of the nodes is properly annotated } \\
\text { Number of the links which can validly connect two nodes }\end{array}$ & 0 & 17 & $5.46(\mathrm{SD}=3.72)$ & 26 \\
$\begin{array}{l}\text { Number of the links which can validly connect two nodes, } \\
\text { but the nodes are not properly annotated }\end{array}$ & 0 & 16 & $4.96(\mathrm{SD}=3.30)$ & 0 \\
$\begin{array}{l}\text { Number of the links which are not properly annotated } \\
\text { Number of cross links which can validly connect two }\end{array}$ & 0 & 21 & $6.39(\mathrm{SD}=3.91)$ & 0 \\
$\quad$ nodes between different clusters & & & $0.14(\mathrm{SD}=0.43)$ & 3 \\
$\begin{array}{l}\text { The score of the associated propositions is properly } \\
\text { clustered }\end{array}$ & 0 & 9 & $4.86(\mathrm{SD}=1.96)$ & 10 \\
$\begin{array}{l}\text { The score of a concept map } \\
\text { Nhe }\end{array}$ & 0 & 86 & $37.28(\mathrm{SD}=19.62)$ & 123 \\
\hline
\end{tabular}


The scores were rated by the teacher and a graduate student. They are familiar with concept mapping and the reading materials. The results are shown in Table 2. In this table, we also present the score of an expert's map. In contrast to the expert map, the quality of the students' maps is not good. The score of the expert's map is triple to the mean score of the students' concept maps. We found several mistakes that the students frequently made.

1. The words in a concept node are more proper as linking words.

2. A concept node was not annotated with concepts, but with propositions.

3. Most of the students cannot find the cross links. Just one group can find two cross links, and four group found one.

4. Linking words cannot validly connect two nodes.

5. We found that many maps are chain structure.

\section{Analysis of the students' use of the system facilities}

To examine how the student uses the system functions to construct the concept maps for the third article, this study analyzed the system logs recorded on database. Table 3 summarizes the analysis. The results showed that 35 groups used text highlight. These groups highlighted 13.06 text segments in average. However, they did not post any comments or questions.

Within the highlighted text segments, 7.63 segments are concepts, 0.5 segments are linking words, and 2.06 segments are propositions in average. These text segments may be used to signal the concepts, linking words, and propositions to support the construction of their concept maps. Therefore, we further analyzed whether text highlight can affect the quality of the students' concept maps. We compared the groups who used text highlight $(\mathrm{G} 1, n=35)$ and the groups who did not use text highlight (G2, $n=34)$. An independent-sample $t$ test was conducted to compare the score of the concept maps of G1 and G2. The result revealed no significant effects of text highlight on concept map score between G1 $($ Mean $=40.94, \mathrm{SD}=19.36)$ and $\mathrm{G} 2$ (Mean $=33.20, \mathrm{SD}=19.44 ; t=1.593$, $p=0.116)$.

Table 3 The analysis of annotation and concept mapping behaviors

\begin{tabular}{lll}
\hline & Mean & SD \\
\hline Number of highlighted text segments $(n=35)$ & 13.06 & 8.47 \\
Number of highlighted text segments are concepts $(n=35)$ & 7.63 & 5.80 \\
Number of highlighted text segments are links $(n=35)$ & 0.5 & 0.9 \\
Number of highlighted text segments are propositions $(n=35)$ & 2.06 & 2.79 \\
Number of Comments $(n=69)$ & 0 & 0 \\
Number of Questions $(n=69)$ & 0 & 0 \\
Number of colors used in a concept map $(n=69)$ & 6.07 & 3.86 \\
\hline
\end{tabular}


In terms of the colors used for their concept maps, most of the students like to use colors for their concept maps. Forty-nine groups have changed the default colors for their concept map. They averagely used six colors in a concept map. These results also reflect that they have the requirements for vivid interface and engaging interaction patterns.

\section{Discussion}

This paper developed a Web-based e-book system with a concept mapping tool. Although we did not evaluate the usability of this system, some evidences can infer that the system is ease of learning and use. First, all the students can use the system to construct a concept map just through watching the $6 \mathrm{~min}$ video. When the students constructed the concept map for the first article, they did not ask us about how to use the system. Second, the students just proposed three functional requirements (4-6) to the second prototype in the questionnaire. These are more advanced functionalities. It represents that the students are satisfied with the basic functionalities of the system.

From the students' feedback, we can find that many suggestions are related to providing more fun and engagingness. In the analysis of the students' use of system facilities, we also found that most of the students like to change the default color and use other colors for their concept maps. These results represent that the students have requirements for engagingness, which is essential for any learning system or learning activity. Therefore, the next system prototype must not only satisfy functional requirements but also provide a more vivid interface and engaging interaction patterns or instructional activities. For example, the system can provide more colors and styles for highlights and nodes. Teachers can also design concept mapping activities with game characteristics.

From the questionnaire, we found that the students had a positive attitude toward concept mapping after using the system in the concept mapping course. They believed that concept mapping is helpful for their learning. They also believed that they are capable of constructing a concept map. However, the intention to use concept mapping was low. The reason for their lack of motivation to use it despite the fact that they believe it is useful for their learning and that they are capable of constructing concept maps needs further research.

By the analysis of the students' concept maps, we found that the quality of their maps is low. The major reason for the low quality is that most of their maps are chain structure. The students constructed their maps according to the order of the information presented in the article; hence, the structure of their maps resembled a chain (Kinchin et al. 2000). Two reasons might explain this result. First, the students did not fully understand how to construct a concept map. They are not capable of identifying concepts and relationship between them. We suggest that students should be trained for a longer duration and provide them more examples and scaffolding support. Furthermore, teachers can instruct students to analyze text structures (Akhondi et al. 2011). Text structures are used to arrange and connect ideas. Therefore, learning text structures may help students to identifying concepts 
and relationship between them in an article. Second, displaying the e-book and the concept mapping tool in the same page has its advantages and drawbacks. The advantage is that the cognitive load is low. Because students can directly refer the reading text when constructing a concept map, they do not need to remember the text and recall when constructing their map. However, the drawback is that students will not try to comprehension the text and just follow the text to construct a concept map. The problem is related to whether the cognitive load is extraneous or germane. Therefore, the question whether displaying the e-book and the concept mapping tool in separated pages is better than in the same page should be examined in our future work.

Text highlight is the most frequently used reading study strategy (Ovsiannikov et al. 1999). However, only half of the students have used text highlight when reading third article. The low use may be explained by two reasons. First, text highlight serves encoding and reviewing roles to support text reading. The third article is short (293 Chinese characters). Therefore, the students did not need text highlight to support them encoding and reviewing text. Second, the e-book and concept mapping system are presented in the same page. The students can directly refer the reading text when constructing a concept map, and they did not need text highlight to support remember and recall text when constructing their map. Within the highlighted text segments, most of the highlighted text segments are the same with the concepts (7.63/13.06), linking words (0.5/13.06), and propositions (2.06/ 13.06) in their concept maps. Therefore, text highlight may support the construction of concept maps. An independent-sample $t$ test found no significant effects of text highlight on concept map score between the groups who used text highlight (G1, $n=35$ ) and the groups who did not use text highlight. Although the results showed no significant effect, further research should be conducted to understand the relationship between text highlight and the behavior and performance of concept mapping.

\section{Conclusion}

This study developed a Web-based e-book with a concept mapping system. A preliminary study which collects users' feedback for the improvement of the system and explore more research issues related to reading and concept mapping was conducted. All the findings were only based on the preliminary study. Several teachers and students' comments about the system and course are proposed. These comments are the base for our next iterative system and course design. We will enhance the system and concept mapping instruction in the next development cycle by providing the following improvements:

1. Provide teachers with an authoring tool that is easy to learn and use for designing concept mapping tasks.

2. Display the e-book and concept mapping interface on separate pages.

3. Provide "undo" and "redo" functions.

4. Enable the nodes in a concept map to align automatically. 
5. Provide the system with a more engaging interface and interaction.

6. Design concept mapping activities that are engaging.

7. Design concept mapping activities that increase students' germane cognitive load.

In addition, several interesting research issues are explored and need to be examined in the future.

1. Why the students lack motivation to use concept mapping despite they believe that it is useful for their learning and that they are capable of constructing concept maps?

2. Whether students with text structure training have better concept mapping performance than the students without text structure training?

3. Whether displaying the e-book and the concept mapping tool in different pages is better for concept mapping than in the same page?

4. Is there any difference in reading or navigational behaviors between the students who use the system displaying the e-book and concept mapping tool in the same page and the students who use the system displaying the e-book and concept mapping tool in separated pages?

Acknowledgments This project was supported by the Ministry of Science and Technology of Taiwanunder contract numbers MOST 102-2811-S-008-010, MOST 103-2811-S-008-005 andMOST 102-2511-S-008-017-MY2.

\section{References}

Akhondi, M., Malayeri, F. A., \& Samad, A. A. (2011). How to teach expository text structure to facilitate reading comprehension. Reading Teacher, 64(5), 368-372.

Al-Khateeb, O. S. M., \& Idrees, M. W. K. (2010). The impact of using KWL strategy on grade ten female students' reading comprehension of religious concepts in Ma'an City. European Journal of Social Sciences, 12(3), 471-489.

Chang, K. E., Sung, Y. T., \& Chen, S. F. (2001). Learning through computer-based concept mapping with scaffolding aid. Journal of Computer Assisted learning, 17(1), 21-33. doi:10.1046/j.1365-2729. 2001.00156.x.

Davies, M. (2011). Concept mapping, mind mapping and argument mapping: What are the differences and do they matter? Higher Education, 62(3), 279-301. doi:10.1007/s10734-010-9387-6.

Hoeft, R. M., Jentsch, F. G., Harper, M. E., I. I. I., Evans, A. W., Bowers, C. A., \& Salas, E. (2003). TPLKATS-concept map: A computerized knowledge assessment tool. Computers in Human Behavior, 19(6), 653-657.

Huang, H. C., Chern, C. L., \& Lin, C. C. (2009). EFL learners' use of online reading strategies and comprehension of texts: An exploratory study. Computers \& Education, 52(1), 13-26. doi:10.1016/j. compedu.2008.06.003.

Kinchin, I. M., Hay, D. B., \& Adams, A. (2000). How a qualitative approach to concept map analysis can be used to aid learning by illustrating patterns of conceptual development. Educational Research, $42(1), 43-57$.

Kintsch, W. (1998). Comprehension: A paradigm for cognition. Cambridge: Cambridge University Press. Kozminsky, E. (2010). Mapping some design consideration for learning from texts. Paper presented at the fourth international conference on concept mapping, Chile. 
Kwon, S. Y., \& Cifuentes, L. (2009). The comparative effect of individually-constructed vs. collaboratively-constructed computer-based concept maps. Computers \& Education, 52(2), 365-375.

Li, L.-Y., Fan, C.-Y., Huang, D.-W., \& Chen, G.-D. (2014). The effects of the e-book system with the reading guidance and the annotation map on the reading performance of college students. Educational Technology \& Society, 17(1), 320-331.

Liu, P. L., Chen, C. J., \& Chang, Y. J. (2010). Effects of a computer-assisted concept mapping learning strategy on EFL college students' English reading comprehension. Computers \& Education, 54(2), 436-445. doi:10.1016/j.compedu.2009.08.027.

Liu, S.-H., \& Lee, G.-G. (2013). Using a concept map knowledge management system to enhance the learning of biology. Computers \& Education, 68, 105-116.

Novak, J. D. (2010). Learning, creating, and using knowledge: Concept maps as facilitative tools in schools and corporations. New York: Taylor \& Francis.

Novak, J. D., \& Cañas, A. J. (2008). The theory underlying concept maps and how to construct and use them (p. 284). Pensacola, FL: Florida Institute for Human and Machine Cognition. www.ihmc.us. http://cmap.ihmc.us/Publications/ResearchPapers/TheoryCmaps/TheoryUnderlyingConceptMaps. htm.

Novak, J. D., \& Gowin, D. B. (1984). Learning how to learn. New York: Cambridge University Press.

Ovsiannikov, I. A., Arbib, M. A., \& McNeill, T. H. (1999). Annotation technology. International Journal of Human-Computer Studies, 50(4), 329-362.

Ponce, H. R., Lopez, M. J., \& Mayer, R. E. (2012). Instructional effectiveness of a computer-supported program for teaching reading comprehension strategies. Computers \& Education, 59(4), 1170-1183.

Tzeng, J.-Y. (2014). Mapping for depth and variety: using a "Six W's" scaffold to facilitate concept mapping for different history concepts with different degrees of freedom. Educational Studies, 40(3), 253-276.

van der Pol, J., Admiraal, W., \& Simons, P. R. J. (2006). The affordance of anchored discussion for the collaborative processing of academic texts. International Journal of Computer-Supported Collaborative Learning, 1(3), 339-357.

Weinerth, K., Koenig, V., Brunner, M., \& Martin, R. (2014). Concept maps: A useful and usable tool for computer-based knowledge assessment? A literature review with a focus on usability. Computers \& Education, 78, 201-209.

Liang-Yi Li is an Assistant Research Scholar in the Research Center for Science and Technology for Learning, National Central University (NCU), Taiwan. His research interests are technology enhanced reading and human-computer interaction. 